\title{
A Convenient Synthesis and Antibacterial Activity of Novel $\alpha$-Aminophosphonic Acid Esters from Amino Acids/Esters (Kabachnik-Fields Reaction)
}

\author{
CH. MOHAN ${ }^{\S}$, B. HARI BABU ${ }^{\S}$, \\ C. NAGA RAJU ${ }^{\S *}$, and R. USHA NAGALAKSHMI \\ ${ }^{\S}$ Department of Chemistry, ${ }^{\#}$ Department of Botany, \\ Sri Venkateswara University, Tirupati-517 502, India. \\ naga_raju04@yahoo.co.in
}

Received 30 September 2007; Accepted 20 November 2007

\begin{abstract}
Synthesis of novel $\alpha$-aminophosphonic acid esters (5a-n) were achieved with high yields through one-pot three component reaction process by Kabachnik-Fields reaction. It involves the reaction among amino acids/esters, substituted aromatic aldehydes and dialkyl phosphites in absolute ethanol at reflux temperature. Their structures were established by elemental analysis IR, ${ }^{1} \mathrm{H},{ }^{13} \mathrm{C},{ }^{31} \mathrm{P}$ NMR and mass spectral data. All the title compounds were screened for their antibacterial activity. Most of the compounds exhibited moderate antimicrobial activity.
\end{abstract}

Keywords: $\alpha$-Amino phosphonic acid esters; Dialkyl phosphates; Aldehydes; Amino acid esters; Antibacterial activity.

\section{Introduction}

Kabachnik-Fields reaction ${ }^{1}$ is used for the synthesis of $\alpha$-amino phosponic acid esters which have useful properties ${ }^{2}$. They have vital role as that of the corresponding amino acid in biological system ${ }^{3-6}$. These are recognized as an important class of enzyme inhibitors either as transition state analogues ${ }^{7}$ or as non-hydrolysable phosphate surrogates ${ }^{8}$ and for the production of catalyst antibodies with esterases or amidase activity ${ }^{9}$. They also find use as potential antibiotics ${ }^{10}$, enzyme inhibitors ${ }^{11}$ and pharmacological agents ${ }^{12}$. $\alpha$-Amino phosphonic acid esters, being phosphorus analogues of amino acid esters ${ }^{13}$ serve as building blocks for peptides. Their commercial applications are enzyme inhibitory neuroactive agents, HIV protease antagonists and collagenase inhibitors. Classical approach to their synthesis is the Kabachnik-Fields reaction, which is a one-pot three component ${ }^{14-16}$ 
operation involving amino acid ester, aldehyde and dialkyl phosphite. To the best of our knowledge, amino acid esters / acids were not used in the synthesis of $\alpha$-amino phosphonates. We have used for the first time successfully and synthesized a series of novel $\alpha$-amino phosphonates containing amino acids / esters under mild conditions without using any catalyst in high yields ${ }^{17}$ Antibacterial activity is also evaluated for the title compounds.

\section{Experimental}

Solvents were used after purifying them by the established procedure. Progress of the reaction and purity of the compounds were monitored by TLC using $n$-hexane and ethylacetate $(1: 1$, by volume) as eluting system on silica gel and iodine as visualizing agent. Melting points were determined in an open capillary tube on Mel-temp apparatus. Micro analysis was performed at CDRI, Lucknow, India. IR spectra were recorded as $\mathrm{KBr}$ pellets on Nicolet 380 double beam spectrophotometer $\left(\bar{v}\right.$ in $\left.\mathrm{cm}^{-1}\right)$ in Environmental Engineering Lab, S.V.University, Tirupati. ${ }^{1} \mathrm{H}$ and ${ }^{13} \mathrm{C}$ NMR spectra were recorded on a Bruker AMX $400 \mathrm{MHz}$ spectrometer operating at $400 \mathrm{MHz}$ for ${ }^{1} \mathrm{H}$ and $100 \mathrm{MHz}$ for ${ }^{13} \mathrm{C}, 161.9 \mathrm{MHz}$ for ${ }^{31} \mathrm{P}$ NMR as solutions in DMSO- $d_{6}$. The ${ }^{1} \mathrm{H}$ and ${ }^{13} \mathrm{C}$ chemical shifts were referenced to tetramethyl silane, and ${ }^{31} \mathrm{P}$ NMR chemical shifts to $85 \% \mathrm{H}_{3} \mathrm{PO}_{4} \cdot{ }^{1} \mathrm{H},{ }^{13} \mathrm{C}$ and ${ }^{31} \mathrm{P}$ NMR spectral data were obtained from Indian Institute of Science, Bangalore, India. Mass spectra were recorded on a Jeol SX 102 DA/600 mass spectrometer using Argon / Xenon $(6 \mathrm{keV}, 10 \mathrm{~mA})$ as the FAB (fast atom bombardment) gas and also a Shimadzu QP-2000 GC-MS instrument.

Synthesis of 2-\{[(diethoxy-phosphoryl)-(4-methoxy-phenyl)-methyl]-amino\} -3-methylbutyric acid methyl ester (4j)

$L$-Valine methyl ester prepared $^{28}(0.838 \mathrm{~g}, 0.005$ mole $)$ and 4-methoxy benzaldehyde $(0.60 \mathrm{~mL}, 0.005$ mole) and diethyl phosphite $(0.64 \mathrm{~mL}, 0.005$ mole $)$ in dry ethanol $(30 \mathrm{~mL})$ was stirred for 30 minutes; then raised to reflux and continued for $5 \mathrm{~h}$. Completion of the reaction was monitored by TLC analysis. After completion of the reaction, solvent was removed in a rota-evaporator. The residue was purified by column chromatography using silica gel (60-120 mesh) as adsorbent and hexane and ethyl acetate (1:1) as an eluent to afford pure $\alpha$-amino phosphonic acid ester $(4 \mathrm{j})$ as a solid, yield $1.43 \mathrm{~g}(74 \%)$, m.p. $116-118^{\circ} \mathrm{C}$.

\section{Results and Discussion}

$\alpha$-Aminophosphonic acid esters (4a-n) were synthesised by one-pot reaction of equimolar quantities of different amino acid esters/acids, substituted aromatic aldehydes and dimethyl / diethyl phosphite in absolute ethanol at reflux temperature for $4-5$ hours in 74-81\% yield (Table 1). Thin layer chromatography (TLC) was employed to monitor reaction progress and to determine the purity of the products. All the title compounds (4a-n) readily dissolve in polar solvents and melted in the range of $69-121^{\circ} \mathrm{C}$ (Scheme 1).

All the compounds (4a-n) showed absorption bands (Table 1) in the region 3200-3390, 1208-1251 and 731-757 $\mathrm{cm}^{-1}$ for $-\mathrm{NH}, \mathrm{P}=\mathrm{O}$ and $\mathrm{P}-\mathrm{C}_{\text {(aliphatic) }}$ respectively ${ }^{18,19}$.

The ${ }^{1} \mathrm{H}$ NMR spectral data ${ }^{20}$ of $4 \mathrm{a}-\mathrm{n}$ are given in Table 2 . The aromatic protons of $\alpha$-amino phosphonic acid esters showed a complex multiplet at $\delta 6.15-8.69$. The P-C-H proton resonated as a multiplet ${ }^{21}$ at $\delta 3.77-4.86$ due to coupling with phosphorus and N-H. The N-H proton signals appeared at $\delta 5.38-6.90$ as doublet $(J=15-7.2 \mathrm{~Hz})$. These signals are confirmed by $\mathrm{D}_{2} \mathrm{O}$ exchange spectral recording. The proton signals of $\mathrm{P}-\mathrm{OCH}_{2}-\mathrm{CH}_{3}$ appeared as a quartet and $\mathrm{P}-\mathrm{OCH}_{2}-\mathrm{CH}_{3}$ gave a triplet at $\delta$ 3.56-3.62 and $\delta$ 1.12-1.19 respectively. 
Table 1. Physical, analytical, infrared and ${ }^{31} \mathrm{P}$ NMR spectral data of 4a-n

\begin{tabular}{|c|c|c|c|c|c|c|c|c|c|c|}
\hline \multirow{2}{*}{ Compd. } & \multirow{2}{*}{ M.P., ${ }^{\circ} \mathrm{C}$} & \multirow{2}{*}{$\begin{array}{c}\text { Yield }^{\mathrm{a}}, \\
\%\end{array}$} & \multirow{2}{*}{$\begin{array}{l}\text { Molecular } \\
\text { formula }\end{array}$} & \multicolumn{3}{|c|}{ Elemental Analysis Found (Calcd), \% } & \multicolumn{3}{|c|}{$\mathrm{IR} \lambda_{\max } \mathrm{cm}^{-1}$} & \multirow{2}{*}{${ }^{31} \mathrm{P} \mathrm{NMR}^{\mathrm{b}}$} \\
\hline & & & & $\mathrm{C}$ & $\mathrm{H}$ & $\mathrm{N}$ & $-\mathrm{NH}$ & $\mathrm{P}=\mathrm{O}$ & P-C $C_{\text {(aliphatic) }}$ & \\
\hline $4 \mathrm{a}$ & $74-76$ & 78 & $\mathrm{C}_{15} \mathrm{H}_{23} \mathrm{~N}_{2} \mathrm{O}_{7} \mathrm{P}$ & $48.09(48.13)$ & $6.15(6.19)$ & $7.40(7.48)$ & 3352 & 1209 & 739 & 19.32 \\
\hline $4 b$ & $69-71$ & 80 & $\mathrm{C}_{17} \mathrm{H}_{27} \mathrm{~N}_{2} \mathrm{O}_{7} \mathrm{P}$ & $50.67(50.74)$ & $6.70(6.76)$ & $6.91(6.96)$ & 3250 & 1208 & 737 & 19.20 \\
\hline $4 c$ & $76-78$ & 76 & $\mathrm{C}_{16} \mathrm{H}_{25} \mathrm{~N}_{2} \mathrm{O}_{7} \mathrm{P}$ & $49.40(49.48)$ & $6.41(6.48)$ & $7.17(7.21)$ & 3259 & 1210 & 752 & 19.60 \\
\hline $4 d$ & $73-75$ & 79 & $\mathrm{C}_{18} \mathrm{H}_{29} \mathrm{~N}_{2} \mathrm{O}_{7} \mathrm{P}$ & $51.84(51.91)$ & $6.95(7.01)$ & $6.67(6.72)$ & 3257 & 1208 & 750 & 19.65 \\
\hline $4 \mathrm{e}$ & $114-116$ & 77 & $\mathrm{C}_{18} \mathrm{H}_{21} \mathrm{~N}_{2} \mathrm{O}_{7} \mathrm{P}$ & $52.87(52.94)$ & $5.14(5.18)$ & $6.79(6.86)$ & 3362 & 1211 & 735 & 19.61 \\
\hline $4 \mathrm{f}$ & $111-113$ & 78 & $\mathrm{C}_{20} \mathrm{H}_{25} \mathrm{~N}_{2} \mathrm{O}_{7} \mathrm{P}$ & $54.96(55.05)$ & $5.72(5.77)$ & $6.38(6.41)$ & 3390 & 1210 & 734 & 19.55 \\
\hline $4 \mathrm{~g}$ & $119-121$ & 80 & $\mathrm{C}_{14} \mathrm{H}_{21} \mathrm{~N}_{2} \mathrm{O}_{7} \mathrm{PS}$ & $42.80(42.85)$ & $5.35(5.39)$ & $7.11(7.13)$ & 3268 & 1212 & 738 & 19.59 \\
\hline $4 \mathrm{~h}$ & $113-115$ & 81 & $\mathrm{C}_{16} \mathrm{H}_{25} \mathrm{~N}_{2} \mathrm{O}_{7} \mathrm{PS}$ & $45.63(45.71)$ & $5.95(5.99)$ & $6.59(6.66)$ & 3269 & 1232 & 731 & 19.52 \\
\hline $4 \mathrm{i}$ & $117-119$ & 75 & $\mathrm{C}_{16} \mathrm{H}_{26} \mathrm{O}_{6} \mathrm{NP}$ & $53.40(53.47)$ & $7.24(7.29)$ & $3.85(3.89)$ & 3220 & 1242 & 752 & 21.52 \\
\hline $4 j$ & $116-118$ & 74 & $\mathrm{C}_{18} \mathrm{H}_{30} \mathrm{O}_{6} \mathrm{NP}$ & $55.85(55.80)$ & $7.76(7.80)$ & $3.58(3.61)$ & 3200 & 1251 & 750 & 21.45 \\
\hline $4 \mathrm{k}$ & $114-116$ & 77 & $\mathrm{C}_{17} \mathrm{H}_{28} \mathrm{O}_{6} \mathrm{NP}$ & $54.76(54.68)$ & $7.49(7.55)$ & $3.70(3.75)$ & 3257 & 1228 & 757 & 21.42 \\
\hline 41 & $115-117$ & 78 & $\mathrm{C}_{19} \mathrm{H}_{32} \mathrm{O}_{6} \mathrm{NP}$ & $56.78(56.84)$ & $7.97(8.03)$ & $3.44(3.48)$ & 3258 & 1229 & 756 & 21.33 \\
\hline $4 \mathrm{~m}$ & $98-100$ & 79 & $\mathrm{C}_{19} \mathrm{H}_{24} \mathrm{O}_{6} \mathrm{NP}$ & $57.95(58.01)$ & $6.10(6.14)$ & $3.52(3.56)$ & 3324 & 1220 & 745 & 22.39 \\
\hline $4 n$ & 99-101 & 80 & $\mathrm{C}_{21} \mathrm{H}_{28} \mathrm{O}_{6} \mathrm{NP}$ & $59.78(59.85)$ & $6.61(6.69)$ & $3.27(3.32)$ & 3325 & 1219 & 736 & 21.97 \\
\hline
\end{tabular}



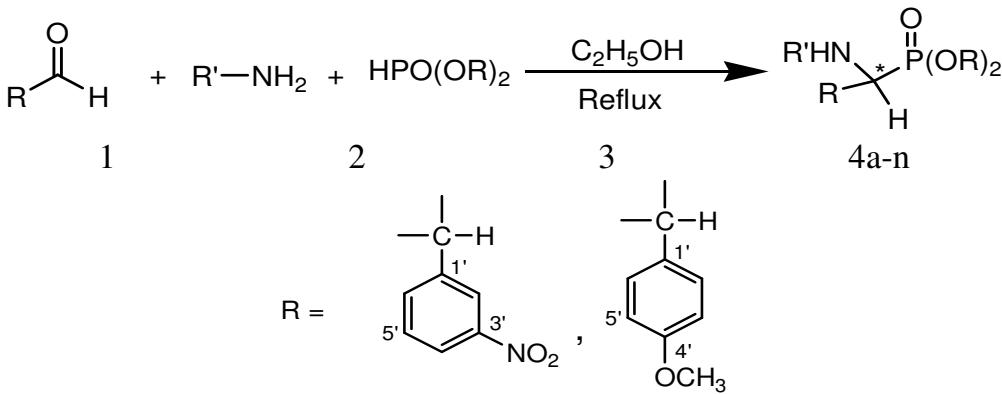

\begin{tabular}{|c|c|c|c|}
\hline Compd. & $\mathrm{R}$ & $\mathrm{R}^{\prime}$ & $(\mathrm{OR})_{2}$ \\
\hline $4 a$ & $3-\mathrm{NO}_{2}-\mathrm{C}_{6} \mathrm{H}_{4}$ & $\left(\mathrm{CH}_{3}\right)_{2}-\mathrm{CH}-\stackrel{\mathrm{I}}{\mathrm{CH}}-\mathrm{COOCH}_{3}$ & $\mathrm{CH}_{3}$ \\
\hline $4 \mathrm{~b}$ & $3-\mathrm{NO}_{2}-\mathrm{C}_{6} \mathrm{H}_{4}$ & $\left(\mathrm{CH}_{3}\right)_{2}-\mathrm{CH}-\stackrel{\mathrm{I}}{\mathrm{C}} \mathrm{H}-\mathrm{COOCH}_{3}$ & $\mathrm{C}_{2} \mathrm{H}_{5}$ \\
\hline $4 \mathrm{c}$ & $3-\mathrm{NO}_{2}-\mathrm{C}_{6} \mathrm{H}_{4}$ & $\mathrm{H}_{3} \mathrm{C}-\left(\mathrm{CH}_{2}\right)_{2}-\mathrm{CH}-\mathrm{COOC}_{2} \mathrm{H}_{5}$ & $\mathrm{CH}_{3}$ \\
\hline $4 d$ & $3-\mathrm{NO}_{2}-\mathrm{C}_{6} \mathrm{H}_{4}$ & $\mathrm{H}_{3} \mathrm{C}-\left(\mathrm{CH}_{2}\right)_{2}-\stackrel{\mathrm{I}}{\mathrm{C}} \mathrm{H}-\mathrm{COOC}_{2} \mathrm{H}_{5}$ & $\mathrm{C}_{2} \mathrm{H}_{5}$ \\
\hline $4 \mathrm{e}$ & $3-\mathrm{NO}_{2}-\mathrm{C}_{6} \mathrm{H}_{4}$ & $\mathrm{C}_{6} \mathrm{H}_{5}-\mathrm{CH}_{2}-\stackrel{\mid}{\mathrm{CH}}-\mathrm{COOH}$ & $\mathrm{CH}_{3}$ \\
\hline $4 \mathrm{f}$ & $3-\mathrm{NO}_{2}-\mathrm{C}_{6} \mathrm{H}_{4}$ & $\mathrm{C}_{6} \mathrm{H}_{5}-\mathrm{CH}_{2}-\stackrel{\mathrm{I}}{\mathrm{C}} \mathrm{H}-\mathrm{COOH}$ & $\mathrm{C}_{2} \mathrm{H}_{5}$ \\
\hline $4 \mathrm{~g}$ & $3-\mathrm{NO}_{2}-\mathrm{C}_{6} \mathrm{H}_{4}$ & $\mathrm{SH}_{3} \mathrm{C}-\mathrm{CH}_{2}-\mathrm{CH}_{2}-\stackrel{\mathrm{I}}{\mathrm{C}}-\mathrm{COOH}$ & $\mathrm{CH}_{3}$ \\
\hline $4 \mathrm{~h}$ & $3-\mathrm{NO}_{2}-\mathrm{C}_{6} \mathrm{H}_{4}$ & $\mathrm{SH}_{3} \mathrm{C}-\mathrm{CH}_{2}-\mathrm{CH}_{2}-\mathrm{CH}-\mathrm{COOH}$ & $\mathrm{C}_{2} \mathrm{H}_{5}$ \\
\hline $4 \mathrm{i}$ & $4-\mathrm{CH}_{3} \mathrm{O}-\mathrm{C}_{6} \mathrm{H}_{4}$ & $\left(\mathrm{CH}_{3}\right)_{2}-\mathrm{CH}-\stackrel{\mathrm{C}}{\mathrm{CH}}-\mathrm{COOCH}_{3}$ & $\mathrm{CH}_{3}$ \\
\hline $4 \mathrm{j}$ & $4-\mathrm{CH}_{3} \mathrm{O}-\mathrm{C}_{6} \mathrm{H}_{4}$ & $\left(\mathrm{CH}_{3}\right)_{2}-\mathrm{CH}-\stackrel{\mathrm{l}}{\mathrm{CH}}-\mathrm{COOCH}_{3}$ & $\mathrm{C}_{2} \mathrm{H}_{5}$ \\
\hline $4 \mathrm{k}$ & $4-\mathrm{CH}_{3} \mathrm{O}-\mathrm{C}_{6} \mathrm{H}_{4}$ & $\mathrm{H}_{3} \mathrm{C}-\left(\mathrm{CH}_{2}\right)_{2}-\stackrel{\mathrm{I}}{\mathrm{C}} \mathrm{H}-\mathrm{COOC}_{2} \mathrm{H}_{5}$ & $\mathrm{CH}_{3}$ \\
\hline 41 & $4-\mathrm{CH}_{3} \mathrm{O}-\mathrm{C}_{6} \mathrm{H}_{4}$ & $\mathrm{H}_{3} \mathrm{C}-\left(\mathrm{CH}_{2}\right)_{2}-\stackrel{\mathrm{I}}{\mathrm{C}} \mathrm{H}-\mathrm{COOC}_{2} \mathrm{H}_{5}$ & $\mathrm{C}_{2} \mathrm{H}_{5}$ \\
\hline $4 \mathrm{~m}$ & $4-\mathrm{CH}_{3} \mathrm{O}-\mathrm{C}_{6} \mathrm{H}_{4}$ & $\mathrm{C}_{6} \mathrm{H}_{5}-\mathrm{CH}_{2}-\stackrel{!}{\mathrm{C}} \mathrm{H}-\mathrm{COOH}$ & $\mathrm{CH}_{3}$ \\
\hline $4 n$ & $4-\mathrm{CH}_{3} \mathrm{O}-\mathrm{C}_{6} \mathrm{H}_{4}$ & $\mathrm{C}_{6} \mathrm{H}_{5}-\mathrm{CH}_{2}-\stackrel{\mathrm{C}}{\mathrm{CH}}-\mathrm{COOH}$ & $\mathrm{C}_{2} \mathrm{H}_{5}$ \\
\hline
\end{tabular}

\section{Scheme 1}

There is corresponding duplication of signals for the ethoxy group in ${ }^{13} \mathrm{C}$ NMR spectra (Table 3). In fact $\underline{\mathrm{CH}}_{3}$ groups resonated as two doublets, one at $\delta 14.8(J=9.2 \mathrm{~Hz})$, and the other doublet at $\delta 14.1(J=9.1 \mathrm{~Hz})$, the $\mathrm{OCH}_{2}$ groups also gave two doublets one at $\delta 62.3(J=7.0 \mathrm{~Hz})$ and the other at $\delta 63.1(J=7.2 \mathrm{~Hz})$. This indicates that the two ethoxy groups which are linked to phosphorus are not magnetically equivalent.

This may be due to restricted rotation at phosphorus centre ${ }^{22}$. The P-C-H chiral carbon gave a doublet in the range of $\delta$ 48.9-51.8 $\left(\mathrm{d}, J_{P C}=143-145 \mathrm{~Hz}\right)$. The methoxy carbon $\quad\left(\mathrm{P}^{-} \mathrm{OCH}_{3}\right)$ resonated as a doublet due to coupling with phosphorus at $\delta 55.0-53.4\left(\mathrm{~d},{ }^{2} J_{P O C}=16.8-16.7 \mathrm{~Hz}\right)$. These values are in agreement with the literature ${ }^{23}$ data ${ }^{31} \mathrm{P}$ NMR chemical shifts ${ }^{24}$ of these compounds (4a-n) appeared in the region19.20-22.29 ppm as expected (Table 1). 
Table 2. ${ }^{1} \mathrm{H}$ NMR Chemical Shifts ${ }^{\mathrm{a}, \mathrm{b}}$ of $4 \mathrm{a}-\mathrm{n}$

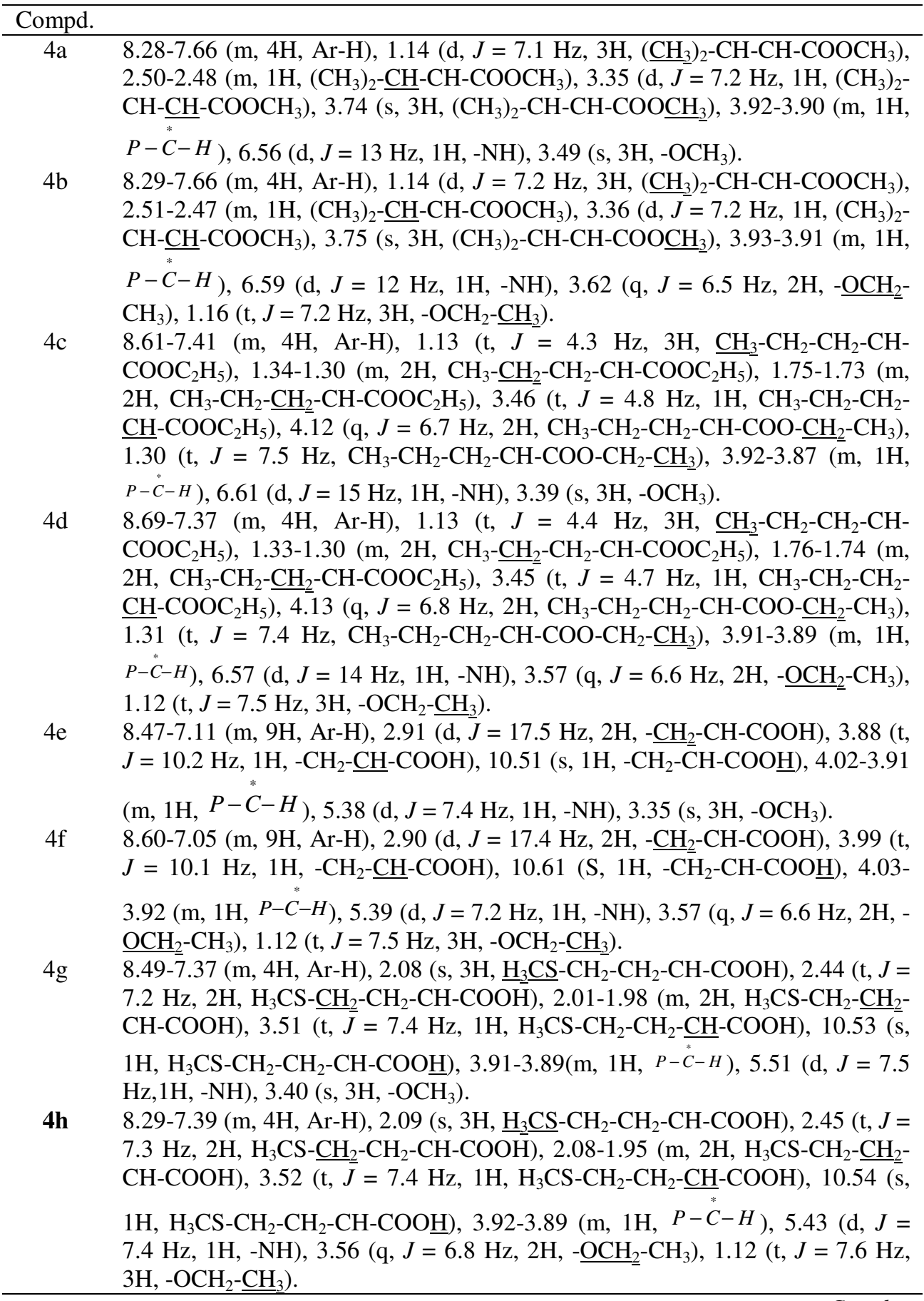


$4 \mathrm{i} \quad 7.52-6.89(\mathrm{~m}, 4 \mathrm{H}, \mathrm{Ar}-\mathrm{H}), 1.12\left(\mathrm{~d}, J=7.2 \mathrm{~Hz}, 3 \mathrm{H},\left(\mathrm{CH}_{3}\right)_{2}-\mathrm{CH}-\mathrm{CH}-\mathrm{COOCH}_{3}\right)$, 2.70-2.68 (m, $\left.1 \mathrm{H},\left(\mathrm{CH}_{3}\right)_{2}-\mathrm{CH}-\mathrm{CH}-\mathrm{COOCH}_{3}\right), 3.44(\mathrm{~d}, J=7.3 \mathrm{~Hz}, 1 \mathrm{H}$, $\left.\left(\mathrm{CH}_{3}\right)_{2}-\mathrm{CH}-\underline{\mathrm{CH}}-\mathrm{COOCH}_{3}\right), 3.74\left(\mathrm{~s}, 3 \mathrm{H},\left(\mathrm{CH}_{3}\right)_{2}-\mathrm{CH}-\mathrm{CH}-\mathrm{COOCH}_{2}\right), 3.91-3.89$ $(\mathrm{m}, 1 \mathrm{H}, P-C-H), 6.08(\mathrm{~d}, J=13.2 \mathrm{~Hz}, 1 \mathrm{H}, \mathrm{NH}), 3.74\left(\mathrm{~s}, 3 \mathrm{H},-\mathrm{OCH}_{3}\right), 3.73(\mathrm{~s}$, $\left.3 \mathrm{H}, \mathrm{Ar}-\mathrm{OCH}_{3}\right)$.

$4 \mathrm{j} \quad 7.34-6.15(\mathrm{~m}, 4 \mathrm{H}, \mathrm{Ar}-\mathrm{H}), 1.14\left(\mathrm{~d}, J=7.2 \mathrm{~Hz}, 3 \mathrm{H},\left(\mathrm{CH}_{3}\right)_{2}-\mathrm{CH}-\mathrm{CH}-\mathrm{COOCH}_{3}\right)$, 2.70-2.67 (m, 1H, $\left.\left(\mathrm{CH}_{3}\right)_{2}-\mathrm{CH}-\mathrm{CH}-\mathrm{COOCH}_{3}\right), 3.35\left(\mathrm{~d}, J=7.4 \mathrm{~Hz}, 1 \mathrm{H},\left(\mathrm{CH}_{3}\right)_{2}-\right.$ $\left.\mathrm{CH}-\mathrm{CH}-\mathrm{COOCH}_{3}\right), 3.75\left(\mathrm{~s}, 3 \mathrm{H},\left(\mathrm{CH}_{3}\right)_{2}-\mathrm{CH}-\mathrm{CH}-\mathrm{COOCH}_{3}\right), 3.93-3.86(\mathrm{~m}, 1 \mathrm{H}$, $P-\stackrel{C}{*}-H), 6.08(\mathrm{~d}, J=13.2 \mathrm{~Hz}, 1 \mathrm{H},-\mathrm{NH}), 3.57\left(\mathrm{q}, J=6.6 \mathrm{~Hz}, 2 \mathrm{H},-\mathrm{OCH}_{2}-\mathrm{CH}_{3}\right)$, $1.18\left(\mathrm{t}, J=6.4 \mathrm{~Hz}, 3 \mathrm{H},-\mathrm{OCH}_{2}-\mathrm{CH}_{3}\right), 3.74\left(\mathrm{~s}, 3 \mathrm{H}, \mathrm{Ar}-\mathrm{OCH}_{3}\right)$.

$4 \mathrm{k} \quad 7.34-6.88(\mathrm{~m}, 4 \mathrm{H}, \mathrm{Ar}-\mathrm{H}), 1.12\left(\mathrm{t}, \mathrm{J}=4.2 \mathrm{~Hz}, 3 \mathrm{H}, \mathrm{CH}_{3}-\mathrm{CH}_{2}-\mathrm{CH}_{2}-\mathrm{CH}-\mathrm{COOC}_{2} \mathrm{H}_{5}\right)$, 1.35-1.32 (m, 2H, $\left.\mathrm{CH}_{3}-\mathrm{CH}_{2}-\mathrm{CH}_{2}-\mathrm{CH}-\mathrm{COOC}_{2} \mathrm{H}_{5}\right)$, 1.76-1.74 (m, 2H, $\mathrm{CH}_{3}-\mathrm{CH}_{2}-$ $\left.\mathrm{CH}_{2}-\mathrm{CH}-\mathrm{COOC}_{2} \mathrm{H}_{5}\right), 3.49\left(\mathrm{t}, \mathrm{J}=4.7 \mathrm{~Hz}, 1 \mathrm{H}, \mathrm{CH}_{3}-\mathrm{CH}_{2}-\mathrm{CH}_{2}-\mathrm{CH}-\mathrm{COOC}_{2} \mathrm{H}_{5}\right.$ ), 4.15 (q, $\left.J=6.8 \mathrm{~Hz}, 2 \mathrm{H}, \mathrm{CH}_{3}-\mathrm{CH}_{2}-\mathrm{CH}_{2}-\mathrm{CH}-\mathrm{COO}-\mathrm{CH}_{2}-\mathrm{CH}_{3}\right), 1.61$ (t, $J=7.4 \mathrm{~Hz}$, $\left.\mathrm{CH}_{3}-\mathrm{CH}_{2}-\mathrm{CH}_{2}-\mathrm{CH}-\mathrm{COO}-\mathrm{CH}_{2}-\mathrm{CH}_{3}\right), 4.85-4.83(\mathrm{~m}, \mathrm{H}, P-\mathrm{C}-\mathrm{H}), 6.90(\mathrm{~d}, J=$ $10.7 \mathrm{~Hz}, 1 \mathrm{H},-\mathrm{NH}), 3.34$ (s, $\left.3 \mathrm{H},-\mathrm{OCH}_{3}\right), 3.74$ (s, 3H, $\left.\mathrm{Ar}-\mathrm{OCH}_{3}\right)$.

$41 \quad 7.34-6.89(\mathrm{~m}, 4 \mathrm{H}, \mathrm{Ar}-\mathrm{H}), 1.11\left(\mathrm{t}, J=4.3 \mathrm{~Hz}, 3 \mathrm{H}, \mathrm{CH}_{3}-\mathrm{CH}_{2}-\mathrm{CH}_{2}-\mathrm{CH}-\right.$ $\mathrm{COOC}_{2} \mathrm{H}_{5}$ ), 1.34-1.31 (m, 2H, $\left.\mathrm{CH}_{3}-\mathrm{CH}_{2}-\mathrm{CH}_{2}-\mathrm{CH}-\mathrm{COOC}_{2} \mathrm{H}_{5}\right), 1.77-1.75$ (m, $\left.2 \mathrm{H}, \mathrm{CH}_{3}-\mathrm{CH}_{2}-\mathrm{CH}_{2}-\mathrm{CH}-\mathrm{COOC}_{2} \mathrm{H}_{5}\right), 3.48\left(\mathrm{t}, \mathrm{J}=4.6 \mathrm{~Hz}, 1 \mathrm{H}, \mathrm{CH}_{3}-\mathrm{CH}_{2}-\mathrm{CH}_{2}-\right.$ $\left.\underline{\mathrm{CH}}-\mathrm{COOC}_{2} \mathrm{H}_{5}\right), 4.14\left(\mathrm{q}, \mathrm{J}=6.9 \mathrm{~Hz}, 2 \mathrm{H}, \mathrm{CH}_{3}-\mathrm{CH}_{2}-\mathrm{CH}_{2}-\mathrm{CH}-\mathrm{COO}-\mathrm{CH}_{2}-\mathrm{CH}_{3}\right.$ ), $1.60\left(\mathrm{t}, J=7.3 \mathrm{~Hz}, 3 \mathrm{H}, \mathrm{CH}_{3}-\mathrm{CH}_{2}-\mathrm{CH}_{2}-\mathrm{CH}-\mathrm{COO}-\mathrm{CH}_{2}-\mathrm{CH}_{3}\right), 4.86-4.83$ (m, H, $P-\dot{C}-H), 6.09(\mathrm{~d}, J=10.8 \mathrm{~Hz}, 1 \mathrm{H},-\mathrm{NH}), 3.56\left(\mathrm{q}, J=6.7 \mathrm{~Hz}, 2 \mathrm{H},-\underline{\mathrm{OCH}_{2}}-\mathrm{CH}_{3}\right)$, $1.19\left(\mathrm{t}, J=7.4 \mathrm{~Hz}, 3 \mathrm{H},-\mathrm{OCH}_{2}-\mathrm{CH}_{3}\right), 3.75\left(\mathrm{~s}, 3 \mathrm{H}, \mathrm{Ar}-\mathrm{OCH}_{3}\right)$.

$4 \mathrm{~m} \quad 7.34-6.75(\mathrm{~m}, 9 \mathrm{H}, \mathrm{Ar}-\mathrm{H}), 2.86\left(\mathrm{~d}, \mathrm{~J}=15.3 \mathrm{~Hz}, 2 \mathrm{H},-\mathrm{CH}_{2}-\mathrm{CH}-\mathrm{COOH}\right), 3.87$ (t, $\left.J=15.6 \mathrm{~Hz}, 1 \mathrm{H},-\mathrm{CH}_{2}-\underline{\mathrm{CH}}-\mathrm{COOH}\right), 10.90\left(\mathrm{~s}, 1 \mathrm{H},-\mathrm{CH}_{2}-\mathrm{CH}-\mathrm{COOH}\right), 3.98-3.91$ $(\mathrm{m}, 1 \mathrm{H}, P-C-H), 6.08(\mathrm{~d}, J=11.3 \mathrm{~Hz}, 1 \mathrm{H},-\mathrm{NH}), 3.37\left(\mathrm{~s}, 3 \mathrm{H},-\mathrm{OCH}_{3}\right), 3.72(\mathrm{~s}$, $\left.3 \mathrm{H}, \mathrm{Ar}-\mathrm{OCH}_{3}\right)$.

4n $\quad 7.35-6.75(\mathrm{~m}, 9 \mathrm{H}, \mathrm{Ar}-\mathrm{H}), 2.89\left(\mathrm{~d}, J=15.4 \mathrm{~Hz}, 2 \mathrm{H},-\mathrm{CH}_{2}-\mathrm{CH}-\mathrm{COOH}\right)$, $3.87\left(\mathrm{t}, J=15.2 \mathrm{~Hz}, 1 \mathrm{H},-\mathrm{CH}_{2}-\underline{\mathrm{CH}}-\mathrm{COOH}\right), 10.89\left(\mathrm{~s}, 1 \mathrm{H},-\mathrm{CH}_{2}-\mathrm{CH}-\right.$ $\mathrm{COOH}), 3.87-3.80\left(\mathrm{~m}, 1 \mathrm{H}, P-{ }^{*}-H\right), 6.09(\mathrm{~d}, J=11.2 \mathrm{~Hz}, 1 \mathrm{H},-\mathrm{NH}), 3.59$ $\left(\mathrm{q}, J=6.8 \mathrm{~Hz}, 2 \mathrm{H},-\mathrm{OCH}_{2}-\mathrm{CH}_{3}\right), 1.17\left(\mathrm{t}, J=7.3 \mathrm{~Hz}, 3 \mathrm{H},-\mathrm{OCH}_{2}-\mathrm{CH}_{3}\right)$, $3.75\left(\mathrm{~s}, 3 \mathrm{H}, \mathrm{Ar}-\mathrm{OCH}_{3}\right)$.

${ }^{a}$ Chemical shifts in ppm from TMS and coupling constants $J(H z)$ in parenthesis ${ }^{b}$ Recorded in DMSO-d . $_{\text {. }}$

In their FAB mass spectra $^{25}$ (Table 4), molecular ions were observed at m/z 409 (28), 394 (43), 385 (31), 402 (17.3) and 421 (9.3) corresponding to the compounds 4e, 4g, 4i, 41 and $4 \mathrm{n}$ respectively.

\section{Antibacterial activity}

Compounds 4a-n were screened for their antibacterial activity against gram positive bacteria, Staphylococcus aureus, Bacillus faecalis and gram negative bacteria, Escherichia coli, Klebsiella pneumoniae by the disc diffusion $\operatorname{method}^{26,27}$ in luria bertani nutrient agar medium at two concentrations $(75,100 \mu \mathrm{g} / \mathrm{mL})$ in DMSO. These solutions containing $10^{6}$ cells / $\mathrm{mL}$ were added to each Whatmann No.1 (made in UK) filter paper disc $(6 \mathrm{~mm}$ diameter) and DMSO was used as the control. The freshly prepared agar medium containing bacteria species was loaded to the discs by using micropipette. The plates were incubated at 
$35^{\circ} \mathrm{C}$ and examined for zone of inhibition around each disc after $24 \mathrm{~h}$. Average value is taken out of three experimental results. The results (Table 5) were compared with the activity of the standard antibiotic Penicillin $(75 \mu \mathrm{g} / \mathrm{mL})$.

Table 3. ${ }^{13} \mathrm{C}$ NMR data ${ }^{\mathrm{a}, \mathrm{b}}$ of $4 \mathrm{e}, 4 \mathrm{~g}, 4 \mathrm{j}$ and $4 \mathrm{k}$

\begin{tabular}{|c|c|}
\hline $\begin{array}{l}\text { Test } \\
\text { compd. }\end{array}$ & Chemical shifts in ppm \\
\hline \multirow[t]{2}{*}{$4 \mathrm{e}$} & $\begin{array}{l}140.5(\mathrm{C}-1), 128.0\left(\mathrm{C}-2 \& \text { C-6), } 128.5(\mathrm{C}-3 \& \mathrm{C}-5), 125.8(\mathrm{C}-4), 37.5\left(-\underline{\mathrm{CH}}_{2}^{-}\right.\right. \\
\text {CH-COOH }), 63.3\left(-\mathrm{CH}_{2}-\underline{\mathrm{CH}}-\mathrm{COOH}\right), 177.8\left(-\mathrm{CH}_{2}-\mathrm{CH}-\underline{\mathrm{COOH}}\right), 48.9(\mathrm{~d}, \mathrm{~J}=143\end{array}$ \\
\hline & $\begin{array}{l}\mathrm{Hz}, 1 \mathrm{C}, P-C H, 138.2\left(\mathrm{C}-1^{\prime}\right), 124.9\left(\mathrm{C}-2^{\prime}\right), 148.3\left(\mathrm{C}-3^{\prime}\right), 122.2\left(\mathrm{C}-4^{\prime}\right), 129.2(\mathrm{C}- \\
\left.5^{\prime}\right), 135.3\left(\mathrm{C}-6^{\prime}\right), 53.4\left(\mathrm{~d}, J=16.8 \mathrm{~Hz}, 1 \mathrm{C},-\mathrm{OCH}_{3}\right) .\end{array}$ \\
\hline \multirow[t]{2}{*}{$4 \mathrm{~g}$} & $\begin{array}{llll}16.3\left(\underline{\mathrm{H}}_{3}-\underline{\mathrm{CS}}-\mathrm{CH}_{2}-\mathrm{CH}_{2}-\mathrm{CH}-\mathrm{COOH}\right), & 29.7\left(\mathrm{H}_{3} \mathrm{CS}-\underline{\mathrm{CH}}_{2}-\mathrm{CH}_{2}-\mathrm{CH}-\mathrm{COOH}\right), \quad 33.5 \\
\left(\mathrm{H}_{3} \mathrm{CS}-\mathrm{CH}_{2}-\mathrm{CH}_{2}-\mathrm{CH}-\mathrm{COOH}\right), 63.5 & \left(\mathrm{H}_{3} \mathrm{CS}-\mathrm{CH}_{2}-\mathrm{CH}_{2}-\underline{\mathrm{CH}}-\mathrm{COOH}\right), 178\left(\mathrm{H}_{3} \mathrm{CS}-\right.\end{array}$ \\
\hline & $\begin{array}{l}\left.\mathrm{CH}_{2}-\mathrm{CH}_{2}-\mathrm{CH}-\mathrm{COOH}\right), 50.2\left(\mathrm{~d}, J=144 \mathrm{~Hz}, 1 \mathrm{C}, P-{ }^{\prime} H\right. \\
\left.2^{\prime}\right), 139.1\left(\mathrm{C}-1^{\prime}\right), 122.9(\mathrm{C}- \\
2^{\prime}, 148.2\left(\mathrm{C}-3^{\prime}\right), 122.9\left(\mathrm{C}-4^{\prime}\right), 129.4\left(\mathrm{C}-5^{\prime}\right), 134.7\left(\mathrm{C}-6^{\prime}\right), 54.5(\mathrm{~d}, J=16.7 \mathrm{~Hz}, \\
\left.1 \mathrm{C},-\mathrm{OCH}_{3}\right) .\end{array}$ \\
\hline \multirow[t]{2}{*}{$4 j$} & 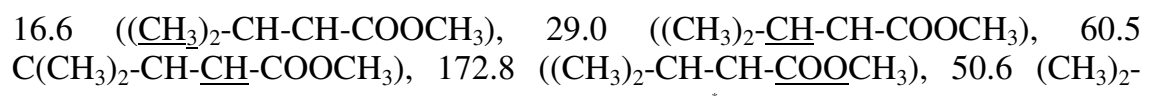 \\
\hline & 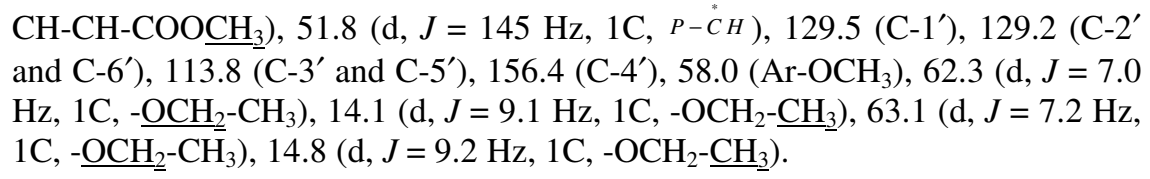 \\
\hline \multirow[t]{2}{*}{$4 \mathrm{k}$} & $\begin{array}{l}13.9\left(\mathrm{CH}_{3}-\left(\mathrm{CH}_{2}\right)_{2}-\mathrm{CH}-\mathrm{COOC}_{2} \mathrm{H}_{5}\right), \quad 16.2\left(\mathrm{CH}_{3}-\underline{\mathrm{CH}_{2}}-\mathrm{CH}_{2}-\mathrm{CH}-\mathrm{COOC}_{2} \mathrm{H}_{5}\right), 34.3 \\
\left(\mathrm{CH}_{3}-\mathrm{CH}_{2}-\underline{\mathrm{CH}_{2}}-\mathrm{CH}-\mathrm{COOC}_{2} \mathrm{H}_{5}\right), 60.1\left(\mathrm{CH}_{3}-\left(\mathrm{CH}_{2}\right)_{2}-\mathrm{CH}-\mathrm{COOC}_{2} \mathrm{H}_{5}\right), 172.1\left(\mathrm{CH}_{3}-\right. \\
\left.\left(\mathrm{CH}_{2}\right)_{2}-\mathrm{CH}-\underline{\mathrm{COOC}}{ }_{2} \mathrm{H}_{5}\right), \quad 62.7 \quad\left(\mathrm{CH}_{3}-\left(\mathrm{CH}_{2}\right)_{2}-\mathrm{CH}-\mathrm{COO} \underline{\mathrm{CH}} \underline{2}_{2}-\mathrm{CH}_{3}\right), \quad 13.8 \quad\left(\mathrm{CH}_{3}-\right.\end{array}$ \\
\hline & $\begin{array}{l}\left.\left(\mathrm{CH}_{2}\right)_{2}-\mathrm{CH}-\mathrm{COO}-\mathrm{CH}_{2}-\mathrm{CH}_{3}\right), 50.1(\mathrm{~d}, J=144 \mathrm{~Hz}, 1 \mathrm{C}, P-\mathrm{CH}), 129.8\left(\mathrm{C}-1^{\prime}\right), \\
128.3\left(\mathrm{C}-2^{\prime} \& \mathrm{C}^{\prime} 6^{\prime}\right), 113.4\left(\mathrm{C}-3^{\prime} \& \mathrm{C}-5^{\prime}\right), 159.2\left(\mathrm{C}-4^{\prime}\right), 63.0\left(\mathrm{Ar}-\mathrm{OCH}_{3}\right), 55(\mathrm{~d}, J \\
\left.=16.8 \mathrm{~Hz}, 1 \mathrm{C},-\mathrm{OCH}_{3}\right) .\end{array}$ \\
\hline
\end{tabular}

${ }^{a}$ Chemical shifts in ppm from TMS and coupling constants $J(H z)$ in parenthesis. ${ }^{b}$ Recorded in DMSO-d .

Table 4. FAB mass spectral data of $4 \mathrm{e}, 4 \mathrm{~g}, 4 \mathrm{j}, 4 \mathrm{l}$ and $4 \mathrm{n}$

\begin{tabular}{cc}
\hline Compd. & \multicolumn{1}{c}{$\mathrm{m} / \mathrm{z},(\%)$} \\
\hline $4 \mathrm{e}$ & $409\left(28.1, \mathrm{M}^{\bullet+}+1\right), 363(43.7), 335(9.3), 288(100), 258(3.1), 242(12.5), 83(28.1)$. \\
$4 \mathrm{~g}$ & $394\left(42.8, \mathrm{M}^{\bullet+}+2\right), 352(7.1), 318(8.9), 207(5.3), 141(100)$. \\
$4 \mathrm{j}$ & $385\left(31.4, \mathrm{M}^{\bullet+}-2\right), 370(20), 334(100), 309(8.5), 238(5.7), 105(12.8)$. \\
41 & $402\left(17.3, \mathrm{M}^{\bullet+}+1\right), 376(13), 316(15.2), 298(8.6), 257(100), 121(21.7)$. \\
$4 \mathrm{n}$ & $421\left(9.3, \mathrm{M}^{\bullet+}\right), 391(7.8), 331(59.3), 307(100), 178(5.2), 102(81.2)$. \\
\hline
\end{tabular}

The compound $4 \mathrm{~h}$ showed higher activity against gram negative bacteria when compared to that of the standard. 4d showed higher activity against Escherichia coli when compared to that of the standard. The compound $4 \mathrm{i}$ exhibited more activity, against gram positive bacterium Staphylococcus aureus when compared to that of the penicillin. Majority of the compounds exhibited promising anti-bacterial activity. 
Table 5. Antibacterial activity ${ }^{\mathrm{a}}$ of new $\alpha$-amino phosphonic acid esters (4a-n)

\begin{tabular}{ccccccccc}
\hline & Staphylococcus aureus & \multicolumn{2}{c}{ Bacillus faecalis } & Escherichia coli & \multicolumn{2}{c}{$\begin{array}{c}\text { Klebsiella } \\
\text { pneumoniae }\end{array}$} \\
\cline { 2 - 8 } Compd. & 75 & 100 & 75 & 100 & 75 & 100 & 75 & 100 \\
& $\mu \mathrm{g} / \mathrm{mL}$ & $\mu \mathrm{g} / \mathrm{mL}$ & $\mu \mathrm{g} / \mathrm{mL}$ & $\mu \mathrm{g} / \mathrm{mL}$ & $\mu \mathrm{g} / \mathrm{mL}$ & $\mu \mathrm{g} / \mathrm{mL}$ & $\mu \mathrm{g} / \mathrm{mL}$ & $\mu \mathrm{g} / \mathrm{mL}$ \\
\hline $4 \mathrm{a}$ & 6 & 6 & 6 & 10 & 7 & 8 & 8 & 9 \\
$4 \mathrm{~b}$ & - & - & 6 & 8 & 9 & 10 & 10 & 11 \\
$4 \mathrm{c}$ & 9 & 11 & 8 & 10 & - & - & 11 & 12 \\
$4 \mathrm{~d}$ & - & - & 8 & 10 & 10 & 11 & 9 & 10 \\
$4 \mathrm{e}$ & 7 & 9 & - & - & 6 & 8 & 6 & 8 \\
$4 \mathrm{f}$ & - & - & 9 & 10 & 8 & 9 & 10 & 11 \\
$4 \mathrm{~g}$ & 8 & 9 & 8 & 10 & 8 & 10 & 6 & 7 \\
$4 \mathrm{~h}$ & 7 & 10 & - & - & 10 & 11 & 12 & 13 \\
$4 \mathrm{i}$ & 11 & 12 & 8 & 9 & 8 & 10 & 7 & 8 \\
$4 \mathrm{j}$ & - & - & 9 & 10 & 8 & 9 & 8 & 9 \\
$4 \mathrm{k}$ & 8 & 9 & 7 & 8 & 9 & 10 & 10 & 11 \\
$4 \mathrm{l}$ & 6 & 8 & 8 & 10 & 9 & 11 & 9 & 10 \\
$4 \mathrm{~m}$ & 7 & 8 & 9 & 10 & 8 & 9 & 8 & 9 \\
$4 \mathrm{n}$ & 8 & 9 & 8 & 10 & 8 & 10 & 9 & 10 \\
Penicillin & 9 & - & 8 & - & 7 & - & 11 & - \\
\hline
\end{tabular}

${ }^{a}$ Concentration in ppm $^{b}$ Standard antibacterial compound

\section{Conclusion}

Synthesis of $\alpha$-amino phosphonic acid esters is achieved in high yields by one-pot three component reaction. Advantages of this synthetic procedure are the operational simplicity high yields and scope as it is applicable to various aldehydes and a variety of amino acids / esters. A few of these compounds exhibited high anti-bacterial activity than the representative compounds.

\section{Acknowledgements}

The authors express thanks to Prof. C. Devendranath Reddy, Sri Venkateswara University, Tirupati, for his encouragement and helpful discussions and the directors of CDRI, Lucknow and SIF, IISc, Bangalore for the elemental analyses and spectral data.

\section{References}

1. (a) Kabachnik M I and Medved T Y, Rok. Akad. Nawk. SSSR, 1952, 689, Chem. Abstr, 1953, 47, 2724. (b) Fields, E.K., J Am Chem Soc., 1952, 74, 1528.

2. Cherkasov R A and Galkin V I, Khim U S P, 1998, 67, 940; Russ Chem Rev., 1998, 67, 857.

3. (a) Gancarz R and Chakraborty S, Synthesis, 1997, 625. (b) Giannousi P P and Bartlett P A, J Med Chem., 1987, 30, 1603.

4. (a) Kajaraski P and Lejczak B, Phosphorus Sulphur, 1991, 63, 193; (b) Kukhar V P and Hudson H R, Amino Phosphonic and Aminophosphinic Acids, John Wiley \& Sons, 2000.

5. Hanessian S and Bennani Y L, Synthesis, 1995, 1272.

6. Griffith E J, Grayson M and Redmore D, In Topics in Phosphorus Chemistry, Ed., Wiley, New York, 1976, Vol.8. 
7. (a) Karanewsky D S, Badia M C, Cushman D W, DeForrest J M, Denjeneka T, Lee V G, Loots M J and Petrillo E W, J Med Chem., 1990, 33, 1459. (b) Morgan B P, Scholtz J M, Ballinger M D, Zipkin I D and Bartlet PA,. J Am Chem Soc., 1991, 113, 297.

8. Biller S A, Forster C, Gorden E M, Harrity T, Scott W A. and Ciosek C P, J Med Chem., 1998, 31, 1869.

9. (a) Jacobs T W, Schultz P G, Powell M and Sugasawara R, J Am Chem Soc., 1987, 109, 2174. (b) Pollack S J, Jacobs J W and Schultz P G, Science, 1986, 234, 1570.

10. Atherton F R, Hassal C H and Lambert R W, J Med Chem., 1987, 30, 1603.

11. Allen M C, Further W, Tock B, Wade R and Wood J M, J Med Chem., 1989, 32, 1652.

12. Hassal C H, In Antibiotics, F.E. Hahn, Ed, Springer Verlag Berlin, 1983, VI, 1-11.

13. (a) Fields S C, Tetrahedron, 1999, 55, 12237 (b) Redmore D, J Org Chem., 1978, 43, 992.

14. Wu J, Sun W, Xia H G and Sun X, Org Biomol Chem., 2006, 4, 1663-1666.

15. Matveera E D, Podrugina T A, Tishkovskaya E V, Tomilova L G and Zefirov N S, Synlett, 2003, 2321-2324.

16. Lee S G, Lee J K, Song C E and Kim D C, Bull Korean Chem Soc., 2002, 23, 667-668.

17. Firouzabadi H, Iranpoor N and Sobhani S, Synthesis, 2004, 2692.

18. Xue-Jun Mu, Mao-Yilei, Jain-Pina Zou and Wei Zhang, Tetrahedron Lett., 2006, 47, $1125-1127$.

19. Thomas L C, The Interpretation of the Infrared Spectra of Organophosphorus Compounds, Heydon and Sons Ltd., London, 1974.

20. Yadav J S, Reddy BVS, Saritha Raj K, Bhaskar Reddy K and Prasad A R, Synthesis, 2001, 15, 2277.

21. Silverstein R M and Webster F X, Spectroscopic identification of organic compounds, $6^{\text {th }}$ Ed., John Wiley \& Sons, New York, 1998.

22. Alina Maly, Bardara Lejczak and Pawel Kafarski, Tetrahedron Asymmetry, 2003, 14, 1019-1024.

23. (a) Fadel W A and Tesson N, Eur. J Org Chem., 2000, 2153; (b) Firouzabadi H, Iranpour $\mathrm{N}$ and Sobhani S, Synthesis, 2004, 16, 2692.

24. (a) Quin L D and Verkade J G, "Phosphorus ${ }^{31}$ P NMR Spectral Properties in Compound Characterization and Structural Analysis" VCH Publishers, New York, 1994. (b) Syam Prasad G, Radhakrishna J, Manjunatha M, Vijaya Sarathi Reddy O, Krishnaiah M, Suresh Reddy C and Puranik V G, Arkivoc, 2007, XIII, 133-141.

25. Keck H, Kuchen W and Mahler H F, Organic Mass Spectrom, 1980, 15, 591.

26. Cruickshank, K.R., Medical Microbiology, A Guide to Diagnosis and Control of Infection, $2^{\text {nd }}$ Ed. E.S. Livingston Ltd., Edinburgh and London, 1968.

27. Beuer A W, Kirby, M M, Sherries J C and Truck A., Am J Clin Pathol., 1969, 45, 493.

28. Ronald G Webb, J Org Chem., 1969, 34, 576. 


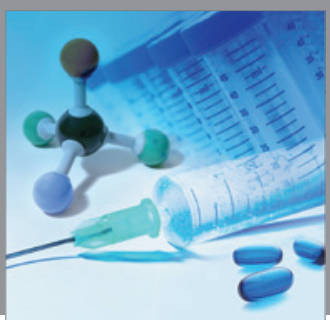

International Journal of

Medicinal Chemistry

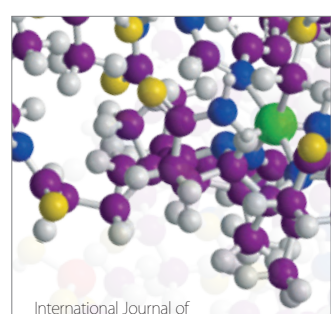

Carbohydrate Chemistry

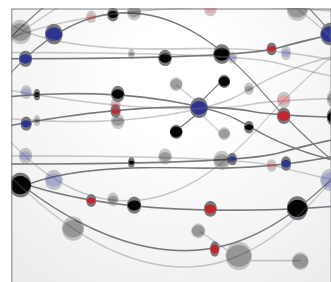

The Scientific World Journal
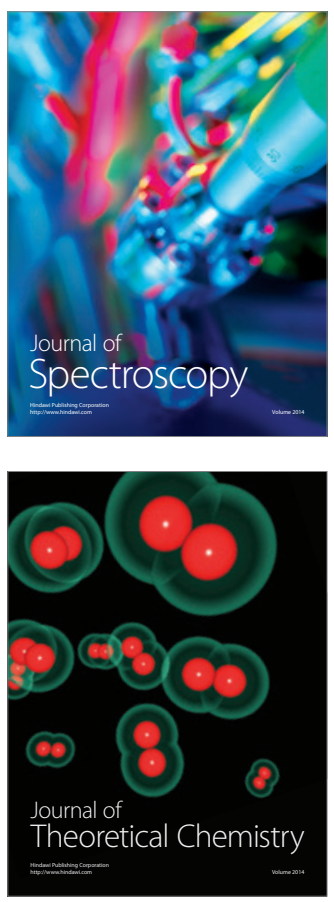
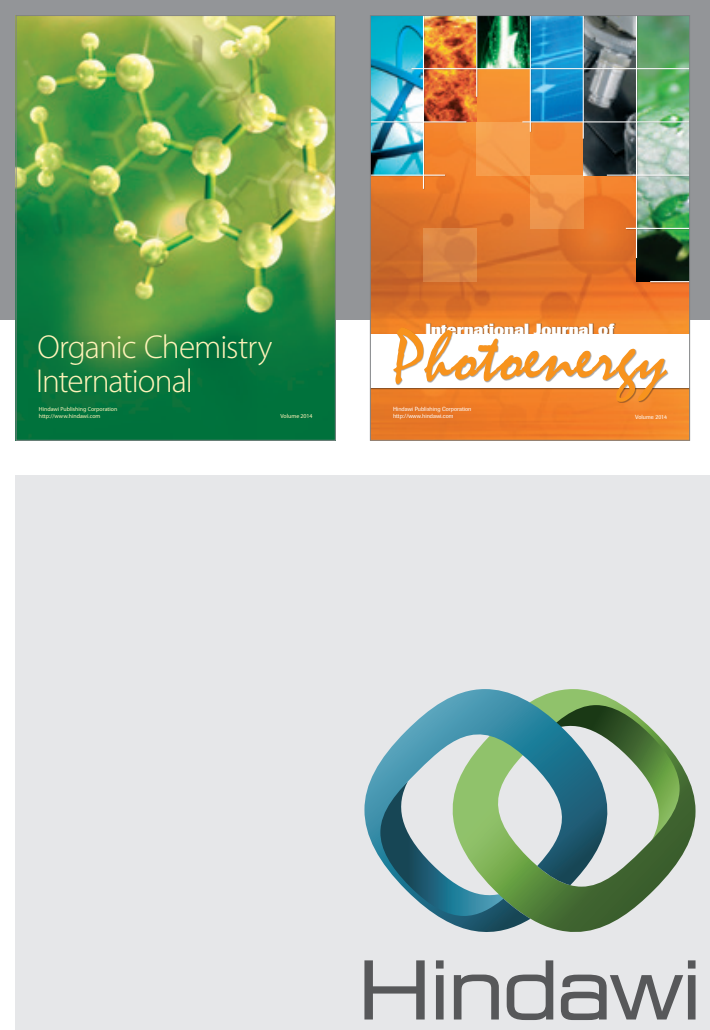

Submit your manuscripts at

http://www.hindawi.com
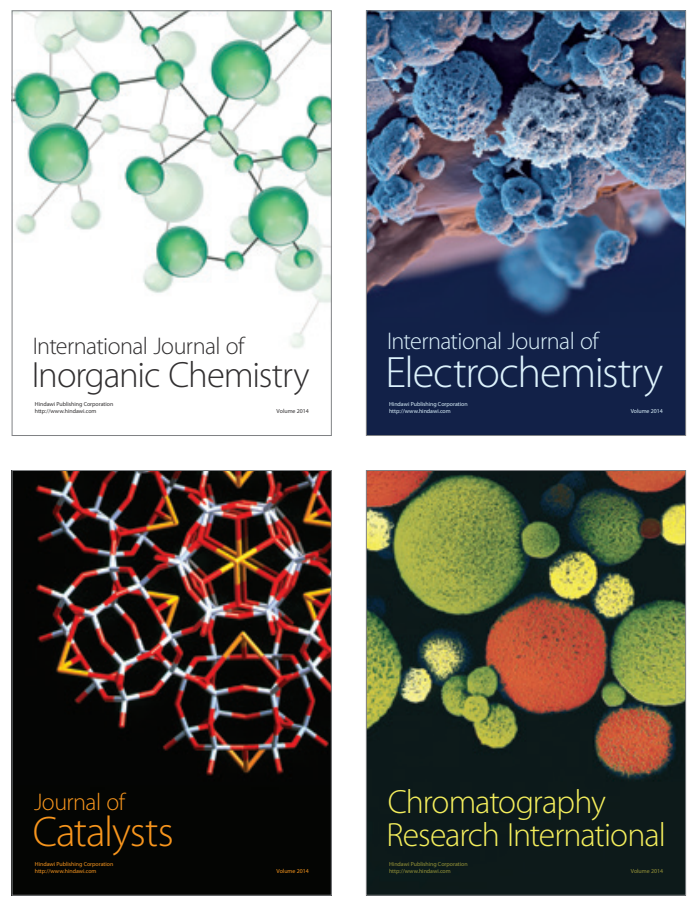
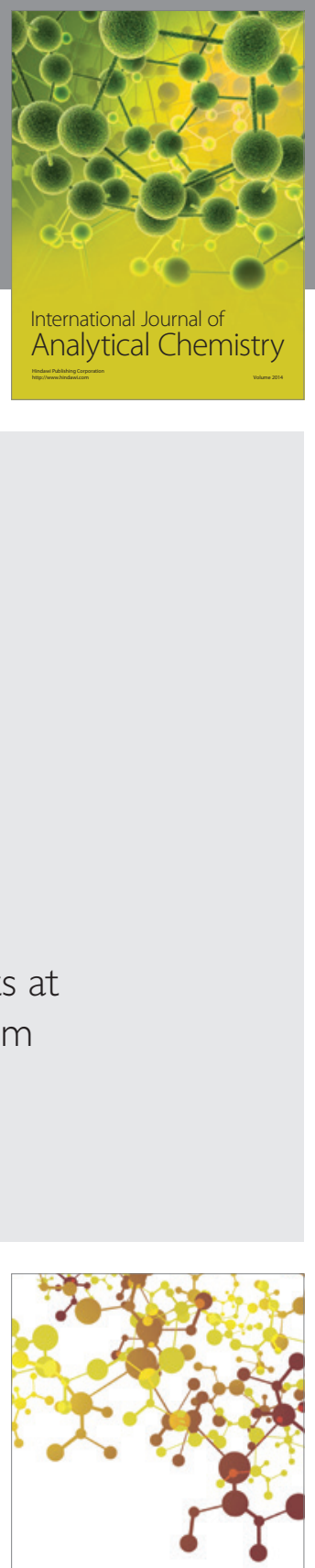

Journal of

Applied Chemistry
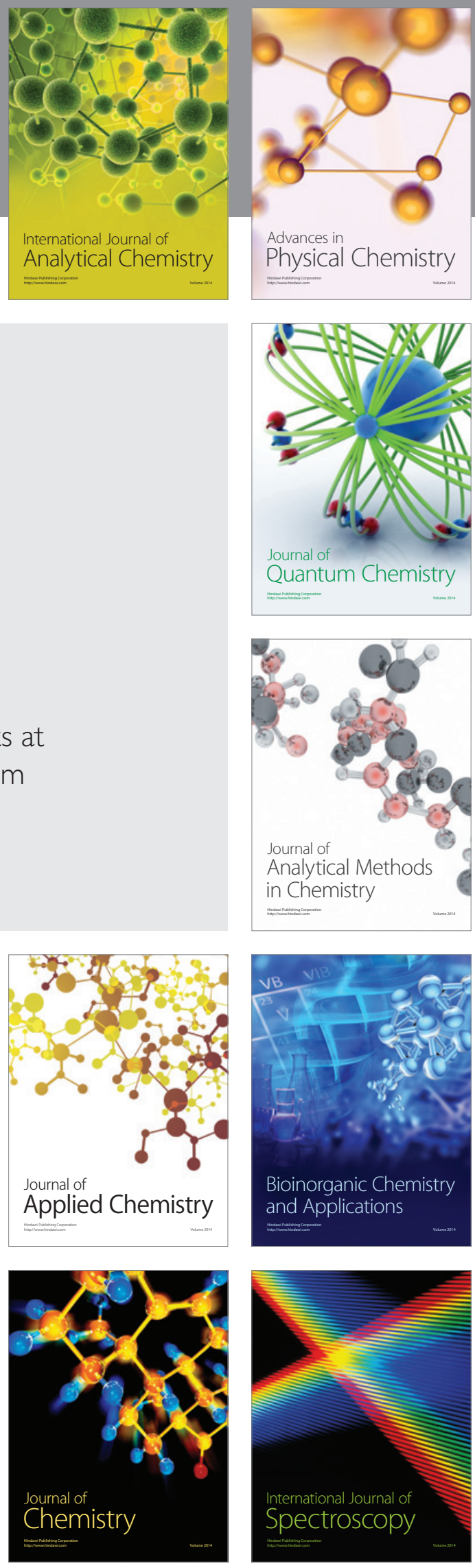